\title{
Differential conditioned suppression in the Konorski-Lawicka paradigm
}

\author{
JOHN MEMMOTT and DOUGLAS REBERG \\ University of Western Ontario, London, Ontario, Canada
}

\begin{abstract}
In conditioned suppression discriminations, the Konorski-Lawicka paradigm involves $\mathrm{A}+$ trials, where conditioned stimulus $\mathrm{A}$ is followed by shock, and $\mathrm{sh} \rightarrow \mathrm{A}-$ trials, where $\mathrm{A}$ is preceded by shock Rats easily mastered this $\mathrm{A}+/ \mathrm{sh} \rightarrow \mathrm{A}-$ discrimination, as indicated by suppression of food-reinforced barpressing on $A+$ trials and acceleration of barpressing on $\mathrm{sh} \rightarrow \mathrm{A}-$ trials. A history of $\mathrm{A}+$ conditioning resulted in nearly perfect discrimination performance on the very first day of $\mathrm{A}+/ \mathrm{sh} \rightarrow \mathrm{A}$ - training, but a history of $\mathrm{sh} \rightarrow \mathrm{A}-$ conditioning retarded development of the discrimination. The basis for the development of the discrimination was discussed in terms of an inferred stimulus $\left(\mathrm{sh}^{\prime}\right)$ arising from the aftereffects of shock.
\end{abstract}

The conditioned inhibition paradigm (Pavlov, 1927 ) is a discrimination training procedure involving two conditioned stimuli, $\mathrm{A}$ and $\mathrm{X}$. On some trials, stimulus $\mathrm{A}$ is presented and followed by an unconditioned stimulus (US). On other trials, $\mathrm{A}$ and $\mathrm{X}$ are presented simultaneously to form a compound stimulus AX, and no US follows. As a result of this procedure (which may be given the notation $\mathbf{A}+/ \mathbf{A X}-)$, conditioned responses are elicited by $\mathbf{A}$ but not by $\mathbf{A X}$. The comparative absence of responding to $\mathbf{A X}$ is generally attributed to inhibitory properties acquired by $X$ which interfere with the ability of $\mathbf{A}$ to elicit the conditioned response (e.g., Rescorla, 1969).

One variation of the above procedure is to present $\mathrm{X}$ and $\mathrm{A}$ as a sequential compound $(\mathrm{X} \rightarrow \mathrm{A}-$ ) on unreinforced trials, where $\mathrm{X}$ immediately precedes $\mathrm{A}$. In this paradigm (which may be given the notation $\mathrm{A}+/ \mathrm{X} \rightarrow \mathrm{A}-$ ), the key to the development of differential responding lies in the occurrence of $X$ prior to unreinforced $A$ trials. Such discriminations appear to be readily acquired. For example, Pavlov (1927, p. 124) reported evidence of differential responding by dogs within a situation in which the sound of a metronome $(\mathrm{A}+)$ was always followed by reinforcement except when preceded by the occurrence of a specific tone $(X \rightarrow A-)$. As Pavlov suggested, it is possible that the "trace of the tone" had combined with the sound of the metronome to function as the effective inhibitory component within this arrangement. The dissipation of the trace prior to termination of $\mathrm{A}$ would remove the effective inhibitory component from the unreinforced compound, and $\mathbf{A}$ would then elicit conditioned responses. Thus, a

This research was sponsored by NRC Grant 9588 to Douglas Reberg and APA Grant 258 to S. B. Kendall. Requests for reprints may be addressed to either author at the Department of Psychology, University of Western Ontario, London, Ontario, Canada N6A 5C2. strong lead stimulus would ensure the continuation of the trace throughout the duration of $A$, thereby rendering to the unreinforced compound its effective inhibitory component.

Pavlov attempted to maximize the effectiveness of the $\mathrm{A}+\mathrm{X} \rightarrow \mathrm{A}$ - paradigm by using particularly intense stimuli (e.g., a "motor car hooter") as X. An interesting variation of this paradigm is the case where the lead stimulus is a US (e.g., Konorski \& Lawicka, 1959; Terry \& Wagner, 1975). For example, in eye-blink conditioning, Terry and Wagner found that rabbits responded more on $\mathrm{A}+$ trials, which consisted of a tone followed by infraorbital shock, than on sh $\rightarrow A-$ trials, which consisted of a tone preceded by shock. This $A+/ s h \rightarrow A-$ discrimination represents one in which the unreinforced component is signaled by the reinforcer at the outset of the trial. One purpose of the present experiment was to determine if Konorski-Lawicka discriminations are also mastered by rats in a conditioned suppression situation where $\mathbf{A}+$ trials are presentations of a conditioned stimulus that terminates with footshock, and $\mathrm{sh} \rightarrow \mathrm{A}$ - trials are unreinforced presentations of a conditioned stimulus immediately preceded by footshock.

Note that the Konorski-Lawicka paradigm actually consists of forward $(\mathrm{A}+)$ and backward $(\mathrm{sh} \rightarrow \mathrm{A}-$ ) conditioning trials. It would seem likely that the development of $\mathbf{A}+/ \mathrm{sh} \rightarrow \mathbf{A}-$ discriminations would be influenced by a prior history of forward or backward conditioning (e.g., Burdick \& James, 1973; Siegel \& Domjan, 1971). We attempted to investigate these possibilities by including groups of subjects exposed to forward and backward conditioning trials prior to receiving $\mathrm{A}+/ \mathrm{sh} \rightarrow \mathrm{A}$ training.

\section{METHOD}

Subjects

Ten male Long-Evans hooded rats with a brief experimental 
history involving appetitive reinforcement served as subjects. They were approximately 150 days old at the start of the experiment and were individually housed with water freely available in their home cages. All subjects were maintained at $80 \%$ of their free-feeding weights throughout the course of the experiment.

\begin{abstract}
Apparatus
Experimental sessions were conducted in a two-lever Lehigh Valley rodent test chamber $(35 \times 27 \times 24 \mathrm{~cm})$. The chamber was housed inside a sound-attenuated cubicle, with an exhaust fan providing ventilation and a masking noise (resting level of $76 \mathrm{~dB}$; re .0002 dynes $/ \mathrm{cm}^{2}$ ). A lever, $2.5 \mathrm{~cm}$ wide, protruded $2 \mathrm{~cm}$ into the right-hand side of the chamber and could be activated by a minimum downward force of $35 \mathrm{~g}(.35 \mathrm{~N})$. The left-hand lever remained retracted throughout the experiment. The floor consisted of 16 stainless steel rods, $.25 \mathrm{~cm}$ in diameter and spaced about $1.5 \mathrm{~cm}$ apart. The unconditioned stimulus (US) was $2-\mathrm{sec}$ 1.5-mA scrambled shock delivered to the grids, walls, and lever of the chamber by a Lehigh Valley shock generator/scrambler. A Mallory sonalert (Model SC628), mounted in the top center of the wall to the left of the response lever, produced a 1-min tone of $86 \mathrm{~dB}$ (re .0002 dynes $/ \mathrm{cm}^{2}$ ), which served as the conditioned stimulus (CS). Standard 45-mg Noyes food pellets were used as reinforcers. Electromechanical programming and recording equipment was housed in an adjoining room.
\end{abstract}

\section{Preliminary Training}

Following magazine and leverpress training, all subjects were allowed to collect 100 food pellets on a continuous reinforcement schedule. During the next two sessions, they were gradually trained to respond on a variable interval (VI) $30-\mathrm{sec}$ schedule with a 5-sec limited hold contingency. Five 42-min experimental sessions were given each week. Training on the VI schedule continued for 15 sessions, by which time visual inspection of the cumulative records revealed within and between session stability for five consecutive sessions. The subjects were then divided into three groups. Groups 1 and 2 had three subjects each. Group 2 originally had four subjects, but one developed an unauthorized escape response to shock early in conditioning and was dropped from the experiment. (Data obtained from this rat were discarded). The groups received $\mathrm{CS}$ habituation and the conditioning procedures as described below.

\section{CS Habituation}

At the close of VI training, each subject received two sessions of preexposure to the CS. During each habituation session, a total of six CSs were delivered, unaccompanied by the US, on a VI 7-min schedule (range: $2-15 \mathrm{~min}$ ). The VI 30-sec reinforcement schedule remained in effect both during and between CS presentations. Inspection of the cumulative records at the end of the habituation sessions indicated that the CS had no appreciable effect on baseline response rate.

\section{Conditioning}

Conditioning proceeded in two phases of 36 sessions each. In Phase 1, subjects in Group 1 received forward conditioning $(A+)$, subjects in Group 2 received backward conditioning $(\mathrm{sh} \rightarrow \mathrm{A}-$ ), and subjects in Group 3 received discrimination training $(\mathbf{A}+/ \mathrm{sh} \rightarrow \mathbf{A}-)$. In Phase 2 , all subjects received $\mathbf{A}+/ \mathrm{sh} \rightarrow \mathbf{A}-$ training.

In all cases, $\mathrm{A}+$ trials consisted of a 1-min presentation of tone immediately followed by a 2 -sec $1.5-\mathrm{mA}$ shock. The sh $\rightarrow \mathrm{A}-$ trials began with a 2 -sec presentation of shock immediately followed by a 1-min presentation of tone that terminated without shock. Six trials were programmed in each VI 30-sec leverpress session. In each session of Phase 1 , Group 1 received six A+ trials and Group 2 received six sh $\rightarrow A$ - trials. For all groups, each session of $A+/ \operatorname{sh} \rightarrow A-$ training included three $A+$ trials and three $s h \rightarrow A-$ trials. Throughout conditioning, trials were presented on the same VI 7-min schedule used during CS habituation.

\section{RESULTS}

The behavioral effects of the CS are reported as suppression ratios computed from the formula $a /(a+b)$, where $a$ is the rate of response during the $\mathrm{CS}$, and $\mathrm{b}$ is the rate during a $1-$ min period immediately preceding the CS. This formula generates a value of .50 when responding is unaffected by the CS. Ratios greater than .50 approaching a maximum of 1.00 indicate acceleration of responding to the $\mathrm{CS}$, and ratios less than .50 approaching a minimum of .00 indicate suppression to the CS. Figure 1 presents the suppression ratios for $\mathrm{A}+$ and $\mathrm{sh} \rightarrow \mathrm{A}-$ trials for subjects in each experimental group averaged over blocks of four sessions during Phases 1 and 2. Illustrative cumulative response records of performance under each conditioning procedure are shown in Figure 2. Suppression ratios will be described separately for each group.

\section{Group 1}

Subjects in Group 1 were exposed to forwardpaired $(\mathrm{A}+)$ trials during Phase 1. As Figure 1 shows, the CS quickly suppressed responding for all subjects in this group when it preceded shock.

During Phase 2, Group 1 subjects received both forward $(\mathrm{A}+)$ and backward $(\mathrm{sh} \rightarrow \mathrm{A}-)$ pairings of the conditioning stimuli. Differential suppression developed very rapidly under the $A+/ s h \rightarrow A-$ conditioning procedure. The difference was evident in the very first session of Phase 2, where ratios on forward conditioning trials averaged .10 and ratios on backward conditioning trials averaged .41 (see record B for R1 in Figure 2). As Phase 2 conditioning continued, the difference between $\mathrm{A}+$ and sh $\rightarrow \mathbf{A}$ - trials increased as acceleration developed on sh $\rightarrow A-$ trials. For all subjects in Group 1, suppression ratios on $\mathrm{sh} \rightarrow \mathrm{A}$ - trials averaged .67 during the last four conditioning sessions of Phase 2. Suppression ratios for $\mathrm{A}+$ trials seemed to be unaffected by the development of acceleration on $\mathrm{sh} \rightarrow \mathrm{A}-$ trials, and averaged .06 for the last four Phase 2 conditioning sessions.

\section{Group 2}

Subjects in Group 2 received backward pairings of the conditioning stimuli (sh $\rightarrow A-$ trials) in Phase 1 . This arrangement resulted in a CS that produced strong acceleration, as indicated by suppression ratios greater than .50 during Blocks 2-9. Ratios increased sharply during early sessions and stabilized at an average level of .83 for the final four Phase 1 sessions.

Phase 2 conditioning under the $A+/ s h \rightarrow A-$ procedure produced additional changes in behavior. Suppression to the CS on A + trials developed very slowly and did not begin to appear until the seventh block of sessions, thereby retarding the development of differential responding within the $A+/ s h \rightarrow A-$ 

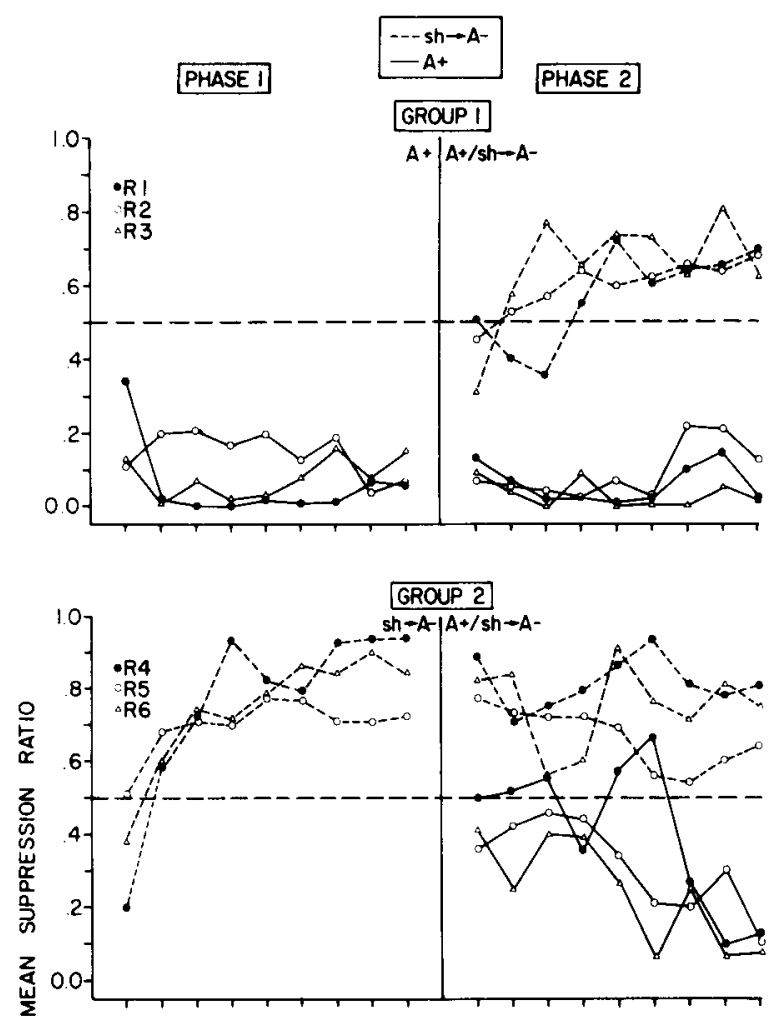

on sh $\rightarrow A-$ trials, acceleration soon developed with continued $A+/ s h \rightarrow A-$ conditioning. During the last four Phase 1 sessions, suppression ratios were greater than .50 in all subjects.

During Phase 2, suppression ratios for $s h \rightarrow A-$ trials continued to rise and reached a mean of .69 during the last four sessions. Almost complete suppression during $\mathbf{A}+$ trials was maintained.

\section{Baseline (Pre-CS) Responding}

In addition to their effects on responding during the CS, the three conditioning procedures greatly disrupted pre-CS baseline responding early in conditioning, resulting in baseline response rates for the first four conditioning sessions that were no more than $22.8 \%$ of preconditioning levels. The degree to which baseline rates recovered by the final four Phase 1 sessions differed among groups. Baseline responding showed the greatest recovery for Group 1 under $\mathbf{A}+$ conditioning (in which all shocks were signaled by the CS), and had returned to $68.2 \%$ of the mean preconditioning response rate. The greatest disruption of baseline responding in Phase 1 occurred in Group 2 under the $s h \rightarrow A-$ procedure

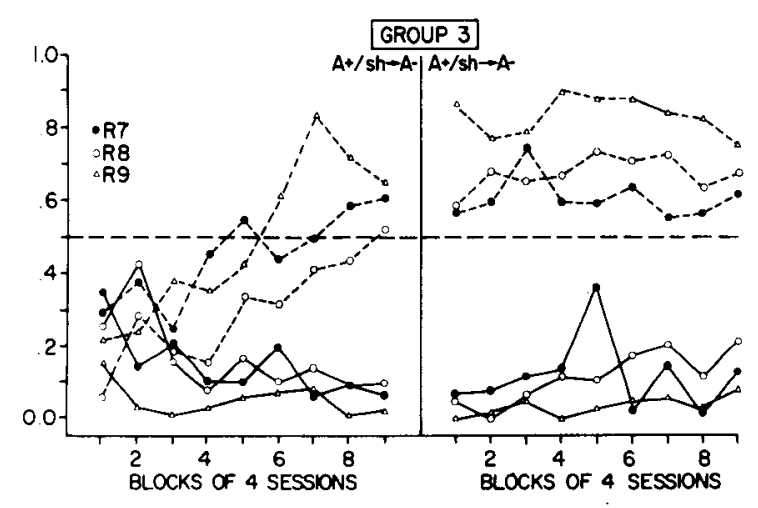

Figure 1. Mean suppression ratios for $\mathbf{A}+$ and $\mathbf{s h} \rightarrow \mathbf{A}-$ trials averaged over blocks of four sessions during Phases 1 and 2 for each experimental subject.

procedure. However, by the close of Phase 2, suppression ratios on $\mathrm{A}+$ trials had reached levels comparable to those obtained for Group 1 subjects during $\mathrm{A}+$-only trials. The introduction of $\mathrm{A}+$ trials resulted in a moderate loss of acceleration to the CS on sh $\rightarrow \mathrm{A}$ - trials.

\section{Group 3}

Group 3 subjects were exposed to the discrimination training procedure in which $\mathrm{A}+$ and $s h \rightarrow \mathrm{A}-$ trials were present from the outset of the experiment. Differential control began to appear by the fourth block of conditioning sessions. Suppression ratios for $\mathbf{A}+$ trials progressively decreased in value and averaged .06 across the final four sessions of Phase 1. Although suppression was initially present

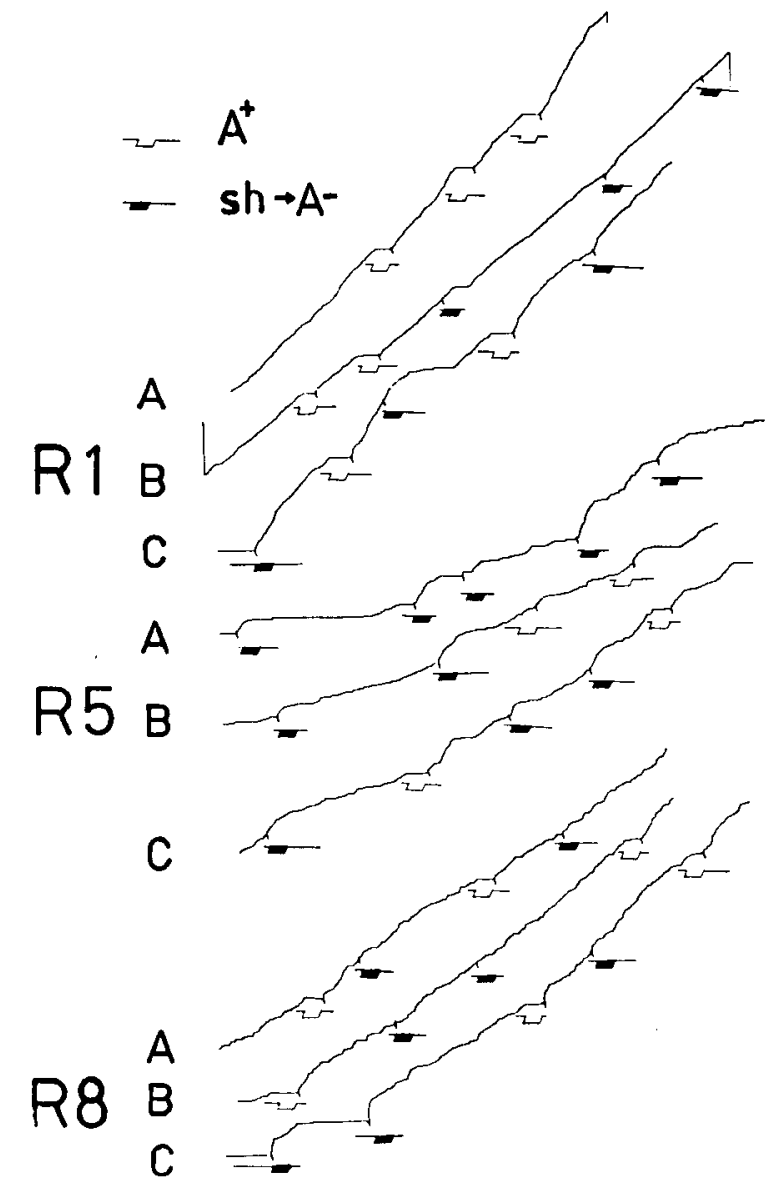

Figure 2. Illustrative cumulative records for subjects in each experimental group obtained during (A) final session of Phase 1 , (B) first session of Phase 2, and (C) final session of Phase 2. Occurrences of shock are indicated by diagonal lines on each record; CS presentations are shown immediately below. 
in which all shocks were unsignaled; the mean baseline response rate under this condition never rose above $8.4 \%$ of its preconditioning value. This result is scarcely surprising in view of the intensely suppressive nature of unsignaled shock observed in previous experiments (e.g., Davis \& McIntire, 1969; Seligman, 1968). The $A+/ s h \rightarrow A-$ discrimination procedure (in which shock was unsignaled only during sh $\rightarrow A$ - trials), resulted in an intermediate level of suppression. Mean baseline rate for Group 3 had recovered to $21.9 \%$ of its preconditioning value during the final Phase 1 conditioning sessions. A similar relationship between percentage of unsignaled shocks and degree of baseline suppression has been observed by Nageishi and Imada (1974).

During Phase 2, all groups were exposed to $\mathbf{A}+/ \mathrm{sh} \rightarrow \mathbf{A}-$ discrimination training. For Group 1 subjects, this resulted in a further reduction in mean baseline responding to $32.7 \%$ of the preconditioning response rate. For Group 2 subjects, however, introduction of the $\mathrm{A}+/ \mathrm{sh} \rightarrow \mathrm{A}$ - procedure was associated with a recovery of baseline responding to $17.4 \%$ of its preconditioning rate. Baseline rate for Group 3 remained fairly stable across experimental phases, averaging $22.4 \%$ of its preconditioning value during the final four sessions of Phase 2.

\section{DISCUSSION}

The present experiment conducted an investigation of differential responding within a conditioned suppression context using the Konorski-Lawicka paradigm. The results may be summarized briefly: (1) Rats were able to acquire a conditioned suppression discrimination in which shock and no-shock trials were reliably signaled by the presence or absence of shock at the outset of each trial. This was a strong and easily established discrimination which involved suppression on $\mathrm{A}+$ trials and acceleration on sh $\rightarrow A-$ trials. (2) It is apparent that a history of $A+$ and $s h \rightarrow A-$ trials has a substantial effect on the development of $\mathbf{A}+/ \mathrm{sh} \rightarrow \mathbf{A}-$ discriminations. (a) Prior $\mathrm{A}+$ conditioning facilitated the development of the discrimination for Group 1 by speeding the elimination of suppression on $\mathrm{sh} \rightarrow \mathrm{A}-$ trials. (b) Prior sh $\rightarrow \mathrm{A}$ - conditioning interfered with the development of the discrimination for Group 2 by retarding the development of suppression on $\mathbf{A}+$ trials.

One might argue that nonassociative mechanisms could contribute to the development of differential suppression in the $\mathrm{A}+/ \mathrm{sh} \rightarrow \mathrm{A}-$ paradigm. For example, shock immediately preceding a CS might disrupt suppression by a "disinhibition" mechanism (e.g., Brimer \& Wickson, 1971), by inducing attacks on the response lever (e.g., Weiss \& Strongman, 1969), by diverting attention from the CS, or by attenuating suppression through a generalization decrement. Although we cannot completely rule out the possible contributions of these nonassociative mechanisms on the basis of this experiment, several considerations suggest that they play a minor role. First, rather low levels of responding are typically induced by nonassociative mechanisms such as disinhibition. Brimer and Wickson (1971), for example, found that shock would disinhibit suppression to a subsequent CS to the extent of 2 or 3 responses/min. As can be seen from the cumulative records in Figure 2 , much higher levels of responding were observed on $s h \rightarrow A-$ trials in the present experiment. Second, informal observations of the subjects' behavior during $\mathrm{sh} \rightarrow \mathrm{A}-$ trials failed to reveal evidence of aggressive reactions to shock. Moreover, subjects were observed consuming reinforcers obtained during the postshock interval, suggesting that postshock responding was food-directed. Finally, experiments that have specifically studied postshock responding have typically found little evidence for an important role of these nonassociative mechanisms (e.g., Burdick \& James, 1973; Imada \& Okamura, 1975).

A more plausible interpretation may be that the aftereffects of shock [which we shall represent as $\mathrm{sh}^{\prime}$ (shock-prime)] have motivational properties that interfere with the ability of the CS to suppress responding. Solomon and Corbit (1973) have developed an opponent process theory of motivation which assumes that an opponent process is elicited by the primary effects of shock. The opponent is assumed to have a relatively long decay time and become stronger as a consequence of repeated exposures to shock. Given that the primary effect of shock is response suppression, the opponent could be reflected in an enhanced rate of responding when shock is terminated. This analysis is similar to the concept of "relaxation" introduced by Denny (1971). $\mathrm{He}$ posits that animals tend to relax following termination of an aversive event, beginning approximately $30 \mathrm{sec}$ after stimulus offset and continuing for about $150 \mathrm{sec}$ thereafter. It is possible that some of the response acceleration appearing at shock offset could be accounted for by an unconditioned tendency to relax which counteracts the suppressive effects of the CS.

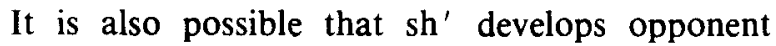
properties as a result of an associative process. As noted above, Pavlov's view of $\mathrm{A}+/ \mathrm{X} \rightarrow \mathrm{A}$ - discriminations (see also Terry \& Wagner, 1975) was that a "trace" of X (or "memorial representation" in Terry and Wagner's terminology) persisted through A to be present when the trial terminated without reinforcement and assumed the role of a conditioned inhibitor. We propose that the "trace" of X be represented as $\mathrm{X}$ ' (read ' $\mathrm{X}$ prime"), and that the $\mathrm{A}+/ \mathrm{X} \rightarrow \mathrm{A}-$ paradigm be fully represented $\mathbf{A}+/ \mathbf{X} \rightarrow \mathbf{A X} \mathbf{X}^{\prime}$. Similarly, $\mathbf{A}+/ \mathrm{sh} \rightarrow \mathbf{A}-$ discrimina- 
tions would be fully represented $\mathrm{A}+/ \mathrm{sh} \rightarrow \mathrm{Ash}^{\prime}-$, where sh' represents the trace aftereffects of shock. It is possible that animals master $\mathbf{A}+/ \mathrm{sh} \rightarrow \mathbf{A}-$ discriminations because sh' is able to acquire inhibitory properties in the manner of an exteroceptive stimulus. Those inhibitory properties, in turn, are presumed to be responsible for the attenuation of suppression on sh $\rightarrow \mathrm{A}-$ trials.

The proposed inhibitory properties of sh' are perhaps most evident in Group 1 where $A+$ training in Phase 1 was followed by virtually complete mastery of the $\mathrm{A}+/ \mathrm{sh} \rightarrow \mathrm{A}$ - discrimination on the first day of Phase 2. Our interpretation of this result is as follows: In Phase 1, a minimum of 2 min intervened between A + trials. Sh' was therefore associated with a period free from shock, and might be expected to acquire inhibitory properties. The attenuation of suppression to $A$ on $\mathrm{sh} \rightarrow \mathrm{A}$ - trials in the first session of Phase 2 could be regarded as evidence for the fear-inhibiting properties sh' developed in Phase 1 (Reberg \& Black, 1969; Rescorla, 1969). A similar interpretation may apply to results obtained by Burdick and James (1973), who found that suppression resulting from $\mathrm{A}+$ trials was virtually eliminated on the very first $\mathrm{sh} \rightarrow \mathrm{A}-$ trial when the procedure was changed to backward conditioning.

Perhaps the least surprising result in this experiment is the finding in Group 2 that backward sh $\rightarrow A-$ training in Phase 1 interfered with the development of the $\mathbf{A}+/ \mathrm{sh} \rightarrow \mathbf{A}$ - discrimination in Phase 2. The interference was due to a marked retardation of the development of suppression on A + trials. Such a result is in agreement with previous research (e.g., Moscovitch \& LoLordo, 1968; Siegel \& Domjan, 1971) demonstrating that backward conditioning generally results in CS developing fear-inhibiting properties which can then interfere with the acquisition of conditioned suppression during subsequent forward conditioning.

At this point, we cannot be certain whether the role of $\mathrm{sh}^{\prime}$ in these discriminations was due to the inherent properties of the opponent process, or if sh' $^{\prime}$ acquires inhibitory properties by signaling a period free from shock. However, the question is open to experimental test. If, indeed, $\mathrm{sh}^{\prime}$ is able to assume an inhibitory function similar to that of an exteroceptive stimulus, that inhibitory function should be evident from other indexes of inhibition such as retarded development of excitation (Rescorla, 1969). It should also be possible to influence excita- tory or inhibitory properties of $s^{\prime}$ by manipulating events in the postshock interval. For example, introducing other stimuli during this period might overshadow sh' and reduce its inhibitory influence. Further examination of the associative properties of the postshock interval and the behavioral influence of sh' seems warranted.

\section{REFERENCES}

Brimer, C. J., \& Wickson. S. Shock frequency, disinhibition. and conditioned suppression. Learning and Motivation, 1971, 2. 124-137.

Burdick, C. K., \& James, J. P. Effects of a backward conditioning procedure following acquisition on extinction of conditioned suppression. Animal Learning \& Behavior, 1973, 1, 137-139.

Davis. H., \& McIntire, R. W. Conditioned suppression under positive, negative, and no contingency between conditioned and unconditioned stimuli. Journal of the Experimental Analysis of Behavior, 1969, 12.633-640.

DENNY, M. R. Relaxion theory and experiments. In F. R. Brush (Ed.), Aversive conditioning and learning. New York: Academic Press, 1971. Pp. 235-295.

Imada, H., \& Okamura. M. Some cues rats can use as predictors of danger and safety. Animal Learning \& Behavior, 1975, 3, 221.225.

Konorskr, J., \& Lawicka, W. Physiological mechanisms of delayed reactions: 1 . The analysis and classification of delayed reactions. Acta Biologica Experimentalis, 1959, 19, 175-197.

Moskovitch, A., \& LoLoRDo, V. M. Role of safety in the Pavlovian backward fear conditioning procedure. Joumal of Comparative and Physiological Psychology, 1968, 66, 673-678.

NAGEISHI, Y., \& IMADA, H. Suppression of licking behavior in rats as a function of predictability of shock and probability of conditioned-stimulus-shock pairings. Journal of Comparative and Physiological Psychology, 1974, 87, 1165-1173.

Pavlov, I. P. Conditioned reflexes (G. V. Anrep, Trans.). New York: Dover, 1960. (Originally published, 1927).

Reberg, D., \& Black, A. H. Compound testing of individually conditioned stimuli as an index of excitatory and inhibitory properties. Psychonomic Science, 1969, 17, 30-31.

Rescorla, R. A. Pavlovian conditioned inhibition. Psychological Bulletin, 1969, 72. 77-94.

Seligman, M. E. P. Chronic fear produced by unpredictable shock. Journal of Comparative and Physiological Psychology, 1968, 66, 402-411.

SIEgEL, S., \& Doman, M. Backward conditioning as an inhibitory procedure. Leaming and Motivation, 1971, 2, 1-11.

Solomon, R. L., \& Corbit, J. D. An opponent-process theory of motivation: II. Cigarette addiction. Journal of Abnormal Psychology, 1973, 81, 158-171.

Terry, W. S., \& WAgner, A. R. Short-term memory for "surprising" versus "expected" unconditioned stimuli in Pavlovian conditioning. Joumal of Experimental Psychology: Animal Behavior Processes, 1975, 104, 122-133.

Weiss, K. M., \& Strongman, K. T. Shock-induced response bursts and suppression. Psychonomic Science, 1969, 15, 238-240.

(Received for publication August 17, 1976; revision accepted December 6, 1976.) 